\title{
Research on the Joint Development of Cities of Guangdong-Hong Kong-Macau Greater Bay Area: A Case Study of Foshan City
}

\author{
Mingyang Luo* \\ Shenzhen Campus, Jinan University, Shenzhen 518000, China \\ *Corresponding author: Mingyang Luo, ibslmy@126.com
}

\begin{abstract}
Promoting the construction of Guangdong-Hong Kong-Macau Greater Bay Area is a major policy decision and national strategy made by the central government of China, which significantly supports the Belt and Road Initiative and maintains the long-term prosperity for the whole country. As an important part of the Guangdong-Hong Kong-Macau Greater Bay Area, Foshan needs to find its own position and direction in order to accelerate the joint development with other cities through five measures.
\end{abstract}

Keywords: Guangdong-Hong Kong-Macau Greater Bay Area; Joint development; City integration; Sanlong Bay innovation cluster; Manufacturing industry innovation

Publication date: October 2021; Online publication: October 25, 2021

\section{Introduction of Guangdong-Hong Kong-Macau Greater Bay Area}

Since the reform and opening-up, especially after the return of Hong Kong and Macau to the motherland, the construction of Guangdong-Hong Kong-Macau Greater Bay Area (GBA) has been intensified. At the same time, the economic strength and regional competitiveness of GBA have been significantly enhanced, leading to the fundamental prerequisites of shouldering GBA as an international first-class bay area and a world-class urban agglomeration established.

The Guangdong-Hong Kong-Macau Greater Bay Area (GBA) comprises of Hong Kong, Macau, and nine cities in Guangdong Province at the Pearl River Delta, including Guangzhou, Shenzhen, Zhuhai, Foshan, Huizhou, Dongguan, Zhongshan, Jiangmen, and Zhaoqing. Its total area measures 56,000 square kilometers with 70 million population ${ }^{[1]}$, which is one of the most open and dynamic regions of China, having an important strategic position in the national development.

\section{Advantages of Guangdong-Hong Kong-Macau Greater Bay Area}

The first is its location. GBA is located in the coastal area of China, which is convenient for transportation and has an important position in the development of the Belt and Road. There are three ports with worldtop throughput: Guangzhou port, Shenzhen port, and Hong Kong International Shipping Center ${ }^{[2]}$. As an international financial, shipping, and trade center, Hong Kong has one of the freest economies in the world with a highly internationalized and legalized environment for business.

Second, its economy is strong. The economic development of the regions covered by the GBA is leading the whole country with a complete industrial system, cluster advantages, and strong economic complementarity. The service industries in Hong Kong and Macau are highly developed; meanwhile, the nine cities at the Pearl River Delta have formed an industrial structure with emerging industries as the forerunner and advanced manufacturing industries as the mainstay. According to the data from the National 
Bureau of Statistics, the total economic volume of GBA in 2019 was about 11.59 trillion yuan, an increase of $6.62 \%$ from $2018^{[3]}$.

Third, it acts as a center to gather innovative elements. Guangdong, Hong Kong, and Macau have outstanding capabilities in technology research. There are a number of famous universities, scientific research institutes, high-tech enterprises, and national science projects, ensuing GBA as an attractive international technology innovation center. In addition, Guangdong Province has actively responded to national policies, implemented the innovation-driven development strategy in depth, and accelerated the establishment of a national innovation demonstration zone. This top-down approach has created favorable innovative conditions for GBA.

\section{Foshan strengthens joint development of cities in GBA}

\subsection{Finding the right position}

From the perspective of political status, Foshan is neither a provincial capital nor a special economic zone. In terms of political status and administrative authority, it is difficult to have the same voice as Guangzhou, Shenzhen, Hong Kong, and Macau; thus, there is a certain gap in resources acquirement. From the perspective of geographical location, Foshan is located at the western part of GBA, connecting the east and the west of China; thus, Foshan has outstanding geographical advantages. The geographical advantage allows Foshan to bring innovation resources from the east to the west, thus expanding the western market with the products and services of GBA. This means that Foshan would not only have the opportunity to access the world's technology, but also seek a bigger market. In terms of gross domestic product (GDP), Foshan is an economically strong city within GBA, ranking only behind Hong Kong, Guangzhou, and Shenzhen (Table 1). It is the fourth city with a GDP exceeding one trillion yuan, higher than other cities in Guangdong Province.

Table 1. GDP data of GBA in 2018 to 2020

\begin{tabular}{cccc}
\hline City & 2018 & 2019 & 2020 \\
\hline Shenzhen & 2.42 & 2.70 & 2.77 \\
Hong Kong & 2.40 & 2.52 & 2.30 \\
Guangzhou & 2.29 & 2.36 & 2.50 \\
Foshan & 0.99 & 1.08 & 1.09 \\
Dongguan & 0.83 & 0.95 & 0.97 \\
Huizhou & 0.41 & 0.42 & 0.42 \\
Macau & 0.36 & 0.37 & 0.35 \\
Zhongshan & 0.36 & 0.31 & 0.32 \\
Zhuhai & 0.29 & 0.34 & 0.35 \\
Jiangmen & 0.29 & 0.31 & 0.32 \\
Zhaoqing & 0.22 & 0.23 & 0.32 \\
Total & 10.87 & 11.59 & 11.70 \\
\hline
\end{tabular}

Data source: National Bureau of Statistics of China ${ }^{[3]}$

Unit: trillion yuan

With the commissioning of Foshan West Railway Station and Shinkansen Airport in addition to the layouts of the Guangdong-Guangxi-Guizhou High-speed Railway Economic Belt Cooperation Pilot Zone as well as other national-level strategic zones, Foshan's geographical and political advantages can be better reflected. Foshan has strong industrial strength, ranking 6th among large and medium-sized cities in the whole country. However, it is not comprehensive to emphasize its role as a manufacturing industry in urban 
development because cultural assets are very important for the influence of cities. In fact, Foshan is one of the birthplaces of Lingnan culture. Cities of the GBA are heavily influenced by Foshan in terms of language, food, folklore, and beliefs, such as the Cantonese opera, Cantonese cuisine, martial arts, and pottery.

\subsection{Measures to accelerate the joint development with other cities}

\subsubsection{Persisting to promote the integration of Guangzhou and Foshan}

First of all, the urban integration of Guangzhou and Foshan is based on the spillover effect of these two cities in the market economy. The increasingly frequent market exchanges from the bottom up have promoted the strategic cooperation between their governments. This is Foshan's political strategy to establish a city network connection with Guangzhou. In terms of transportation tracks, Foshan's government should continue to promote the connection of subway lines, the construction of sea-crossing bridges, and the use of Guangfo Line from Foshan West Railway Station to Guangzhou South Railway Station.

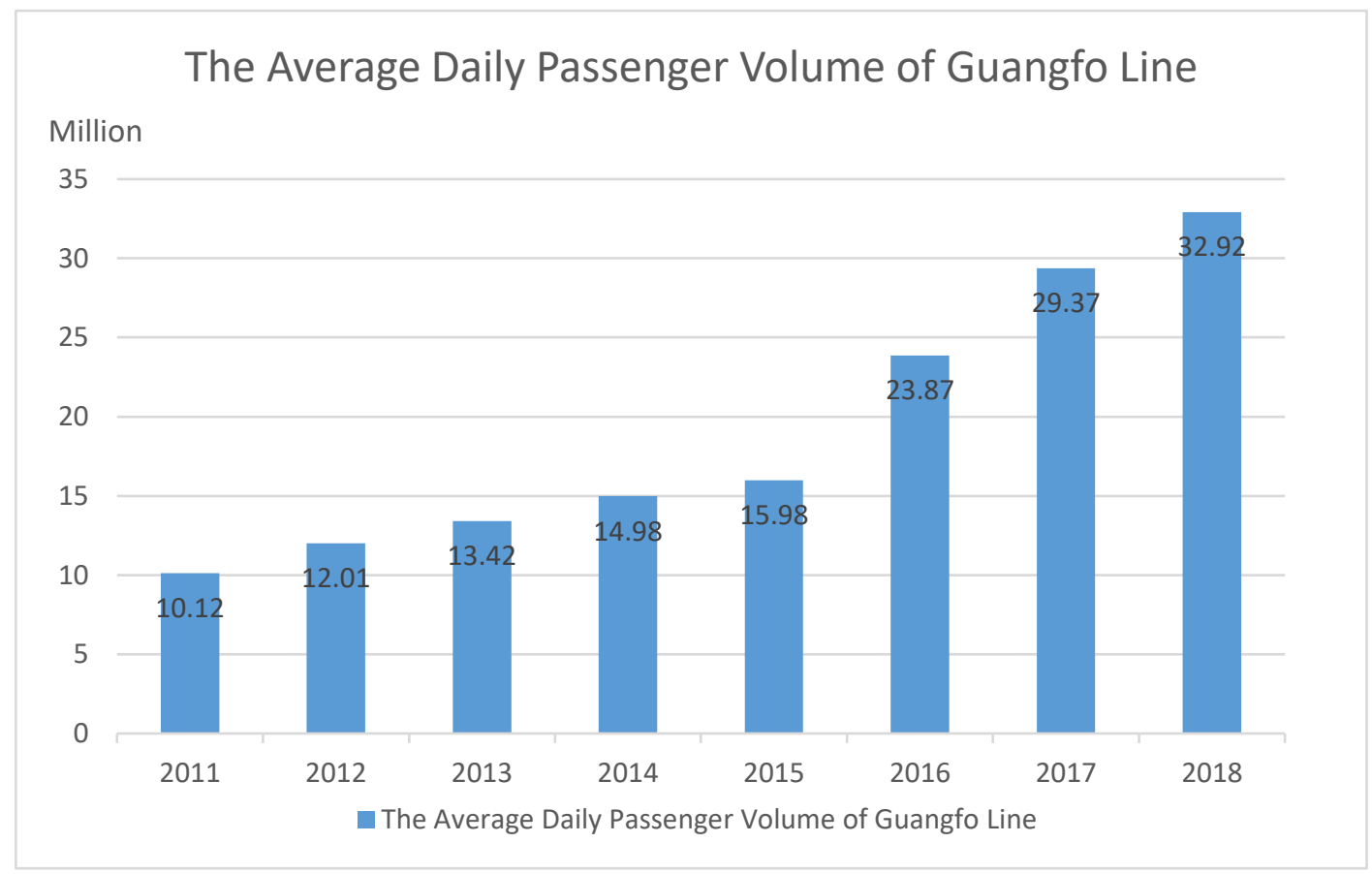

Figure 1. Average daily passenger volume of Guangfo Line 


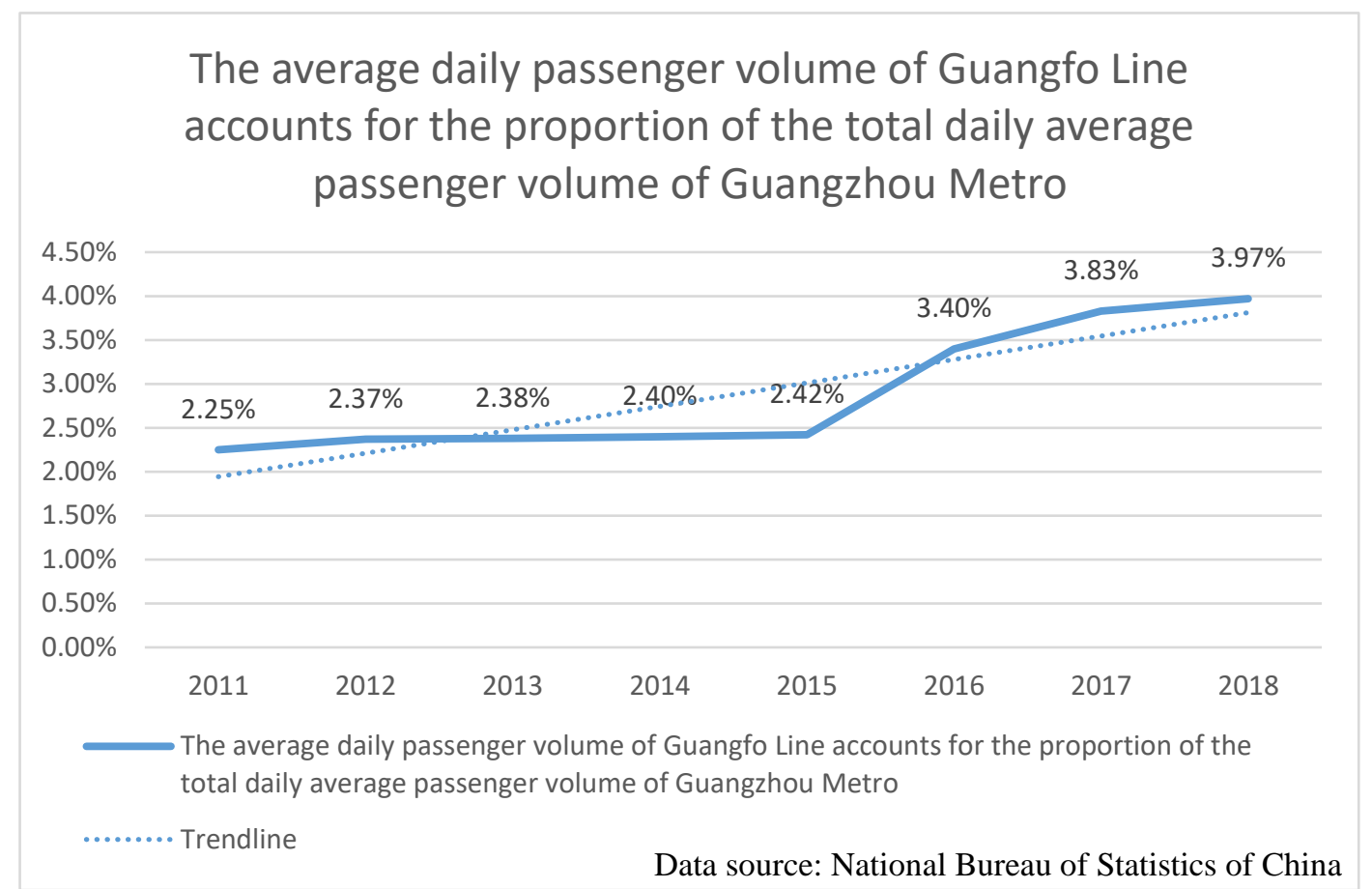

Figure 2. Average daily passenger volume of Guangfo Line accounts for the proportion of the total daily average passenger volume of Guangzhou Metro

The bar chart and line chart above illustrate the average daily passenger volume of Guangfo Line and the average daily passenger volume of Guangfo Line accounting for the proportion of the total daily average passenger volume of Guangzhou Metro, respectively (Figure 1 and Figure 2) ${ }^{[4]}$. The average passenger volume of both the Guangfo Line and Guangzhou Metro have been increasing gradually every year, especially after 2015. This shows that Guangfo Line is beneficial for the integration of Guangzhou and Foshan.

Second, industry complementarity should be placed as an importance. At present, Guangzhou and Foshan have negotiated a joint development of advanced manufacturing parks, including equipment manufacturing, automobiles, new-generation information technology, biomedicine, and health care industry, with a scale of 4 trillion yuan. This would enhance the industrial competitiveness of both cities and expand more industry complementary information.

Third, Foshan can establish a high-quality integration pilot area around the boundary of Guangzhou and Foshan. At present, the pilot area of Guangzhou South Railway Station-Foshan Sanlong Bay-Liwan Hailong Area has taken shape. In view of that, Foshan's government should continually provide policy support and invest funds as well as make effort to build it into a landmark project of Guangfo cooperation as much as possible ${ }^{[5]}$. Due to the advantage of geographical proximity, the trend of integration of Guangzhou and Foshan has blurred the boundary between these two cities. This provides a foundation for the establishment of Foshan's cross border network, which would not only benefit the development of these two cities, but also the progress of GBA.

\subsubsection{Linking Foshan's manufacturing with Shenzhen's innovation}

Shenzhen is another important interface for Foshan to enhance its innovation capabilities. With policy support, Foshan can seize favorable opportunities in establishing major infrastructures, such as the Shenzhen-Zhongshan Bridge and the Hong Kong-Zhuhai-Macau Bridge, make full use of its own advantages in manufacturing and construction, and then actively collaborate with Shenzhen's innovative 
elements, including technology, capital, talents, etc ${ }^{[6]}$. Foshan ought to understand that it can greatly improve the quality of its manufacturing industry by undertaking Shenzhen's innovation spillover effects and industrial cooperation. In this process, Foshan can hold more trade fairs, investment promotion meetings, or set up liaison offices in Shenzhen so as to create more carriers for Foshan's manufacturing and Shenzhen's innovation. Shenzhen (Foshan) Industrial Liaison Office which was established by the Foshan Chancheng District Government in Nanshan District, Shenzhen is a successful case. In addition to that, the district government also successfully held a high-tech industry cooperation meeting.

\subsubsection{Making full use of the advanced service industries in Hong Kong and Macau}

Hong Kong has a mature financial system and service industry experience. The joint development of Hong Kong has established a network relationship between Foshan and Hong Kong. It is one of the earlier strategies that Foshan implemented after joining the GBA. The direction of cooperation with Hong Kong can develop toward Hong Kong finance + Foshan manufacturing, Hong Kong R\&D (research and development) + Foshan innovation, Hong Kong service + Foshan trade, Hong Kong creativity + Foshan culture, and Hong Kong health preservation + Foshan ecological environment ${ }^{[7]}$. Besides, there is a need to further explore a normalized communication, negotiation, and exchange mechanism with Hong Kong in order to help Foshan present its products and services to the world.

At present, the ties between Foshan and Macau are relatively weak; thus, their cooperation mechanism is not yet sound. Therefore, Foshan can learn from the experience of cooperating with Hong Kong to jointly develop with Macau as well. Macau has different culture from the mainland because of some historical reasons. In view of that, Macau can introduce their Macau-Portuguese-style culture and some time-honored brands to consumers in Guangdong Province. This project may attract investors, and with that, Foshan would be able to make full use of the capital, brands, and rich operating experience of Macau to explore new ways of development. Another important thing is encouraging teenagers from Hong Kong and Macau, or overseas students, to settle down by making supportive policies and business-friendly environment. It is an effective method to create a good platform for the gathering of talents, information, technology, and other resources, which would be helpful to build a bridge between Guangdong, Hong Kong, and Macau markets.

\subsubsection{Taking advantage of its geographical location}

Foshan West Railway Station is a significant station of Guiguang and Nanguang high-speed railways. The operation of these two high-speed railways has become the main route connecting Southwest and South China in the new era. The greatly improved traffic conditions have promoted the establishment of Guangdong, Guangxi, and Guizhou high-speed railway economic zone. Furthermore, Guangdong has made Foshan West Railway Station as a center in the Guangdong-Guangxi-Guizhou High-speed Railway Economic Belt Pilot Zone (Guangdong Park), enabling Foshan to establish cross-scale networks and cooperative relations with Guangxi Province and Guizhou Province. Alike Sanlong Bay, Foshan West Railway Station Hub New City is also one of the key geographic carriers for Foshan's integration into GBA. The stations can be used to develop more exhibitions and business offices. With that, the government can then orderly promote the transfer of high-quality resources from GBA to Guangxi and Guizhou region, including advanced industries, technologies, and talents. At the same time, it can attract high-end elements from regions along the Guangdong-Guangxi-Guizhou High-speed Railway Economic Belt gathering in the GBA, such as private investment conferences, food exhibition expos, and the tourism industry alliance of these three provinces. 
Foshan should seize the opportunity to expand the influence of local products and services as well as break through the boundaries divided by political entities through network connection and stronger liquidity. It will not only promote cross-regional cooperation, but also help Foshan accelerate its integration into GBA. In the process of regional cooperation, industrial transfer is the most common way to establish a relationship network. For example, famous ceramic companies in Foshan have begun to move to Wuzhou City (Guangxi), where high-quality and abundant raw materials can be found to further explore the industrial model of "Foshan R\&D and Guangxi Production." Foshan considers the Guangdong-GuangxiGuizhou High-speed Railway Economic Belt Pilot Zone as a huge potential market through cross-regional cooperation in providing multiple opportunities for Foshan to expand its commodity and capital flows, thus further promoting the flow of factors and industrial transfer in the Pearl River Delta region. Following that, it would then be possible to form an industrial cooperation system with gradual development, reasonable division, and complementary advantages, which can benefit the leading role of GBA as well as promote the accelerated development of the surrounding areas.

\subsubsection{Persisting in the construction of Sanlong Bay innovation cluster}

The establishment of the high-end innovation cluster in Sanlong Bay should be continued, thus creating the innovation leader of Foshan. Sanlong Bay is the base of Guangzhou-Foshan co-urbanization, Hong Kong, Macau, and Jihua laboratory (a provincial high-end science and technology innovation platform of Guangdong Province). It is also a regional carrier for Foshan in linking scientific and technological innovation elements from Guangzhou, Shenzhen, and Hong Kong. This is profitable for importing scientific and technological achievements. Sanlong Bay, as a selective space for Foshan's government, forms an urban network by establishing partnerships with governments representing different cities. The establishment and maintenance of these partnerships are actually the collection of resources. Therefore, Sanlong Bay has become a platform for absorbing innovative resources and the engine which leads Foshan in its innovation ${ }^{[8]}$. In order to cope with the new round of development needs, Foshan should reorganize its loose resources and the urban space, further improve its ability to coordinate regional development and gather resource elements, as well as cultivate new local growth poles to maintain its competitive advantage.

\section{Conclusion}

In conclusion, promoting the construction of Guangdong-Hong Kong-Macau Greater Bay Area is to establish a world-class city cluster, which would enrich the connotation of the "One Country, Two Systems" policy, further intensify communication and cooperation among the mainland, Hong Kong, and Macau, as well as maintain the long-term prosperity and stability of Hong Kong and Macau. The construction of GBA can build a new platform for international economic cooperation, further deepen the reform and openingup of China, as well as promote the Belt and Road Initiative and the 21st Century Maritime Silk Road.

As an important part in the construction of the Guangdong-Hong Kong-Macau Greater Bay Area, Foshan needs to strengthen its joint development with other cities. Firstly, Foshan should use this highquality platform to find its own position in the GBA; meanwhile, it should reasonably digest the spillover effects of Guangzhou and Shenzhen, which requires Foshan's manufacturing to actively collaborate with the innovation of Guangzhou, Shenzhen, and Hong Kong. Secondly, Foshan should use the advantage of its location to introduce high-tech achievements from the GBA to the western region of China. Lastly, Foshan needs to persist in the establishment of Sanlong Bay innovation cluster in order to extensively attract local and foreign talents as well as funds. This would provide a new momentum for Foshan and eventually contribute to the construction of the Guangdong-Hong Kong-Macau Greater Bay Area. 


\section{Disclosure statement}

The author declares that there is no conflict of interest.

\section{References}

[1] Constitutional and Mainland Affairs Bureau, 2018, Greater Bay Area Cities. Greater Bay Area, https://www.bayarea.gov.hk/sc/home/index.html

[2] Xie H, Zhang Y, Chen Y, et al., 2021, A Case Study of Development and Utilization of Urban Underground Space in Shenzhen and the Guangdong-Hong Kong-Macao Greater Bay Area. Tunnelling and Underground Space Technology incorporating Trenchless Technology Research, 107.

[3] Data Source: GDP of Cities of Guangdong Province. National Bureau of Statistics. http://www.stats.gov.cn (updated March 2021).

[4] Data Source: Statistics of Subway Passengers in Guangzhou. National Bureau of Statistics. http://stats.gd.gov.cn (updated October 2020).

[5] Ma H, Li Y, Huang X, 2020, Proximity and the Evolving Knowledge Polycentricity of Megalopolitan Science: Evidence from China's Guangdong-Hong Kong-Macao Greater Bay Area, 1990-2016. Journal Indexing \& Metrics, 58(12): 2405-2423.

[6] Wu M, Wu J, Zang C, 2020, A Comprehensive Evaluation of the Eco-carrying Capacity and Green Economy in the Guangdong-Hong Kong-Macao Greater Bay Area, China. Journal of Cleaner Production, 281: 124945.

[7] Ma J, Jiang F, Gu L, et al., 2020, Patterns of the Network of Cross-border University Research Collaboration in the Guangdong-Hong Kong-Macau Greater Bay Area. Sustainability, 12(17): 6846.

[8] Qi T, 2020, Research on the Economic Development of Real Estate in Guangdong-Hong Kong-Macao Greater Bay Area. American Journal of Industrial and Business Management, 10(10). 